\title{
MEMORY AND THE EXPERIENCE OF TIME: CONSIDERATIONS OF A NEUROPHYSIOLOGIST
}

\section{Fabio Benfenati}

\author{
Chair of Human Physiology, Department of Experimental Medicine, \\ University of Genova Medical School and Department of Neuroscience and Brain Technologies, \\ Fondazione Istituto Italiano di Tecnologia, Genova, Italy
}

\begin{abstract}
Learning and memory are fundamental higher brain functions that allow individuals to adapt to the environment, to build up their own history as unique creatures, to widen the personal cultural background and, ultimately, the population culture. The molecular and cellular mechanisms that contribute to short- and long-term memory are extremely conserved across evolution from mollusks to man and among various forms of memory and consist in short-to-long lived rearrangements in synaptic efficiency and in the structure of neuronal networks.
\end{abstract}

Key words: synapse, plasticty, learning, memory consolidation, forgetting, future.

\section{INTRODUCTION}

"To feel today what one felt yesterday isn't to feel - it's to remember today what was felt yesterday, to be today's living corpse of what yesterday was lived and lost"

Fernando Pessoa (1888-1935)

Memory is commonly seen as a positive ability of the individual to improve performance, indispensable for survival and social success. Forgetting, on the other hand, generally has a negative connotation, which is often associated with pathological states and/or aging. This common view is also reflected in our knowledge of the underlying biological processes. While thousands of papers have elucidated the processes of learning and memory from the molecular and cellular level up to the cognitive and psychological level, relatively few data are available on the mechanisms of forgetting.

Memory and forgetting are daily processes of life, which allow us to select from our billions of experiences those that are the most relevant for our personal history and our culture. One could say that without forgetting, memory would be completely useless. This concept is very well exemplified by the short fantasy story "Funes el memorioso" by Jorge Luis Borges (1942). Describing Funes, Borges says: "On falling from the horse, he lost consciousness; when he recovered it, the present was almost intolerable it was so rich and bright; the same was true of the most ancient and most trivial memories. ... And now, his perception and his memory were infallible.... Without effort, he had learned English, French, Portuguese, Latin. I suspect, nevertheless, that he was not very capable of thought. To think is to forget a difference, to generalize, to abstract. In the overly replete world of Funes there were nothing but details, almost contiguous details. ... It occurred to me that each one of my words (each one of my gestures) would live on in his implacable memory; I was benumbed by the fear of multiplying superfluous gestures." The inability to forget details prevents the process of generalization that is necessary for abstract thought and ultimately for making sense of our experiences.

\section{THE ROOTS OF LEARNING AND MEMORY ARE AT THE SYNAPSES}

"All the psychological matters that we are progressively formulating, will have to rely, one day, on an organic substrate" S. Freud, Entwurf einer Psychologie, 1895

The major and most distinctive feature of the nervous system is its astonishing ability to adapt to the environment and to improve its performance over time and experience. In 1906 this special/unique property, collectively named "plasticity", was precisely defined by Santiago Ramon y Cajal as "the property by virtue of which sustained functional changes occur in particular neuronal systems following the administration of appropriate environmental stimuli or the combination of different stimuli". Since the neural changes evoked by the stimuli can persist for a very long time, virtually for the whole life of the individual, neural plasticity could represent an attractive basis for learning and memory. Conversely, the built-in property of 
neural plasticity might allow experience to functionally and structurally shape the nervous system.

The first assembly of neuronal networks is driven by genetic factors, i.e. by the size of the physiological targets and the expression of chemotactic and/or cell adhesion "recognition" proteins whose genes are specifically transcribed and translated by the various neuronal populations [1]. After this first gene-driven developmental period, neuronal circuits are continuously modified and shaped by experience (epigenetic development): synaptic connections that are scarcely used become weaker and weaker and eventually disappear, whereas synapses that are heavily used become stronger and stronger and eventually increase in number. Synaptic strength can be finely tuned over a short or even a long time scale by a combination of factors including previous activity of the network, generation of second messengers, functional changes in pre- and post-synaptic proteins as well as regulation of the expression of genes implicated in growth, survival and synaptic transmission. This results in changes in the efficiency of synaptic transmission that can last from a fraction of a second to minutes in the case of short-term synaptic plasticity (facilitation or depression) to hours, days and months in the case of long-term synaptic plasticity (long-term potentiation, long-term depression). These changes profoundly affect the processing carried out between input and output information and, ultimately, filter and shape the flow of information within the neural network.

Interestingly, after the cornerstone discoveries of Camillo Golgi and Santiago Ramon y Cajal, but some 50 years before Donald Hebb (1949) formulated the idea of synaptic plasticity as the basis of psychological functions [2], Sigmund Freud proposed in his Entwurf einer
Psychologie (1895) that the physical structure of memories consists of a long-lasting activity-dependent modification of information transfer between neurons [3-5]. Freud drew attention to the synapse, which he called "contact barrier", and to the quantity of information " $Q \eta$ " that passes through the synapse during the process of neural excitation, i.e. the equivalent of synaptic strength (Figure 1). He identified two types of communication, that of "permeable or $\varphi$ neurons that behave as if they have no contact barriers", (i.e. neurons which transfer information across the synapse without resistance) and that of "impermeabile or $\psi$ neurons which act in such a way as to permit only a difficult or partial passage of $Q \eta^{\prime \prime}$. Thus, the activity of a network depends on the mosaic of facilitated and nonfacilitated barriers since, as Freud says, Q $\eta$ in an $\alpha$ neuron will be directed toward a more facilitated barrier... and the higher $Q \eta$ during the course of excitation, the greater the facilitation". Thus, memories can be represented as sequences of activity patterns distributed across a population of neurons, which in turn are associated with a different subsequent pattern of encoding. During retrieval, a memory cue may cause neural activity to evolve toward one of these activity patterns [6].

\section{FORGETFULNESS AND MEMORY CONSOLIDATION}

"I've seen things you people wouldn't believe. Attack ships on fire off the shoulder of Orion. I watched C-beams glitter in the dark near the Tannhauser gate. All those moments will be lost in time, like tears in rain."

Ridley Scott, Blade Runner, 1982

Learning induces cellular and molecular changes that
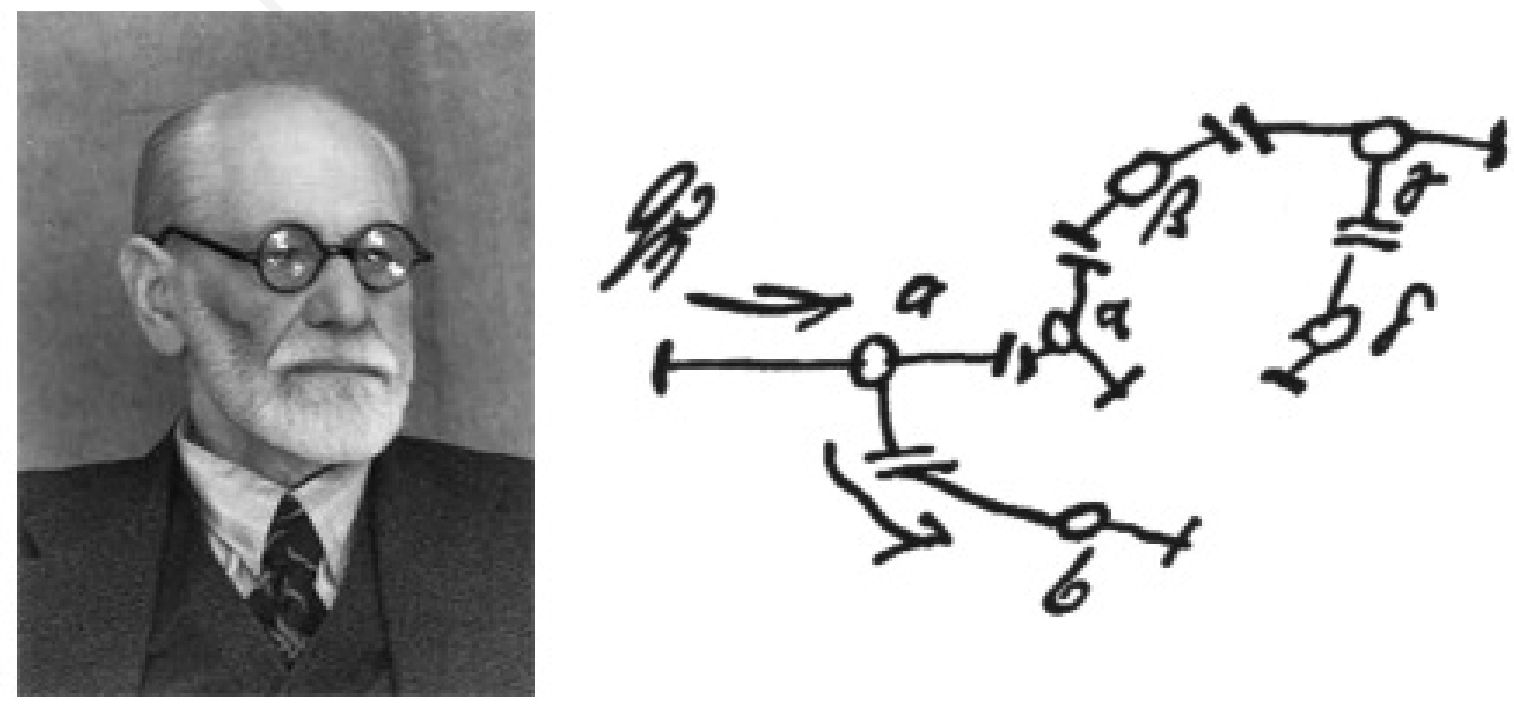

Figure 1. Sigmund Freud and his model drawing of a neural network in which changes in the gain of synaptic connections ("permeability") among neurons is predicted to be the basis of learning and memory. 
facilitate or impair communication among neurons and are fundamental for memory storage. If learning brings about changes in "synaptic strength" within neuronal circuits, the persistence of these changes represents the way memories are stored. Short-term memory is believed to involve only functional changes in pre-existing neuronal networks mediated by a fine-tuning of multiple intracellular signal transductions systems. These short-lived changes can undergo either of two processes: either fade out with time (forgetfulness) or be reinforced and transformed into long-term memory by a process called memory consolidation. Forgetfulness is at least as important as consolidation. Since only a minimal part of what we perceive is useful, the brain needs a mechanism to prevent itself from being burdened by negligible information. To be consolidated, functional changes have to be followed by gene transcription and protein synthesis that produce permanent phenotypic changes in the neuron associated with structural rearrangements in neuronal networks. Thus, memory consolidation is abolished by blocking the synthesis of mRNAs and proteins. Consolidation is not a high fidelity process: stored memories gradually change and fade with time and only the most relevant and useful aspects are retained $[7,8]$.

Several molecular actors and biochemical processes underlie short-term memory processes. Two processes that appear to be a final common pathway are phosphorylation and dephosphorylation of synaptic proteins $[9,10]$. Phosphorylation has dramatic effects on proteins' conformation, interactions and functions. Thus, the balance between phosphorylation and dephosphorylation is tightly regulated in neurons via the activation of kinase and phosphatase enzymes by specific intracellular signaling molecules called "second messengers" that include cyclic AMP and $\mathrm{Ca}^{2+}$. The finely tuned balance between phosphorylation and dephosphorylation has a strong impact on synaptic transmission. Since the activation of the molecules involved in these signaling pathways can last for minutes, it represents a sort of short-term "molecular memory" which can operate as a "push-pull" mechanism playing a key role in regulating the delicate balance between storage and disposal of short-term memories [8,9,11-13].

These purely functional changes cannot survive for long times in the absence of a structural rearrangement of the neurons participating in the modulated synapse. The sustained activation of the same pathways promotes memory consolidation by affecting gene transcription and translation. Sustained stimulation leads to persistent activation of kinase pathways that modulate the activity of transcription/repression factors. The CREB family of transcription regulators is highly conserved across evolution and represents the major switch involved in the transformation of short-term memory into long-term memory. The CREB target genes, whose transcription is regulated during consolidation, include a set of immediate-early genes (such as C/EBP or zif268) that affect transcription of downstream genes. This results in changes, either increases or decreases, in the expression of an array of proteins involved in protein synthesis, axon growth, synaptic structure and function [7,14,15]. When synaptic strength has to be permanently potentiated (long-term potentiation, LTP), ribosomal proteins, neurotrophins, $\mathrm{Ca}^{2+}$-binding proteins, proteins involved in the exo-endocytic cycle of synaptic vesicles and neurotransmitter receptors become upregulated, whereas cell adhesion molecules that usually maintain synaptic stability become downregulated. These specific changes in protein expression favor growth of terminal axon branches and establishments of novel synaptic contacts. Opposite phenomena are believed to occur in the case of longterm depression (LTD) of synaptic strength, favoring a decrease in the number of synaptic connections and/or a decreased activity of the existing synapses.

These properties were remarkably emphasized by Donald Hebb who wrote: "when an axon of cell $A$ is near enough to excite cell $B$ or repeatedly or consistently takes part in firing it, some growth or metabolic change takes place in one or both cells such that A's efficiency, as one of the cells firing $B$, is increased" $[2,4]$. In other words, a "synaptic learning rule" exists by which synapses learn from the pattern of afferent stimulation and persistently change synaptic strength accordingly. Although LTP and LTD were originally referred to specific synapses (in the hippocampus and the cerebellum, respectively), we now know that virtually every synapse can tune its strength by entering a potentiated or depressed state that can last for long periods of time.

\section{DISTINCT FORMS OF MEMORY RELY ON SIMILAR CELLULAR MECHANISMS}

"Without the memories of what was pleasant or unpleasant, it is not possible to be happy, sad, anguished, one cannot get angry, infatuated. One could say that an organism is a memory that acts."

Henri Laborit (1914-1995)

Two major types of memory exist, one for skills and one for knowledge. The first one refers to information storage to perform various reflexive or perceptual tasks and is also referred to as non-declarative or implicit memory because it is recalled unconsciously. The second form of memory, called declarative or explicit memory, because it is recalled by a deliberate and conscious effort, concerns factual knowledge of persons, things, notions and places. Declarative memory can be further subdivided in episodic or autobiographic memory and semantic memory.

Neuropsychological studies, pioneered by the Canadian neuropsychologist Brenda Milner, have shown that the multiple memory systems involve distinct 
brain areas and exhibit distinctive features. Thus, explicit memory requires an intact medial temporal lobe (hippocampus), while implicit memory systems are integrated at various levels in the central nervous system including reflex pathways, striatum, cerebellum, amygdala and neocortex. Moreover, the kinetics of the learning, consolidation and recall phases of memories is quite different. Implicit memory, e.g. learning to ride a bike, takes time and many attempts to build up, while explicit memory, such as learning a page of history or a telephone number, is more immediate and implies a smaller effort. However, while explicit memory fades relatively rapidly in the absence of recall and refreshing, implicit memory is much more robust and may last for all our life even in the absence of further practice $[7,16]$.

The simplest paradigms of implicit memory are elementary forms of non-associative and associative behaviors, which are present in primitive animals. These paradigms have been effectively studied in mollusks, particularly the sea snail Aplysia californica, which has a very simple central nervous system made up of a few thousands neurons (the human brain in comparison is made up of about $10^{11}-10^{12}$ neurons). Aplysia is able to learn specific behaviors that, upon practice, can be consolidated into long-term memories. The animal progressively learns to respond more weakly to repeated innocuous stimuli (e.g. a light tactile stimulus), a behavior called habituation, and to reinforce the response to repeated noxious stimuli (e.g. a painful electrical shock), a behavior known as sensitization. In both cases, the synaptic efficiency in the integration centre of a sensory-motor reflex is changed by experience, leading to an increased response of the reflex in the case of sensitization or to a reflex inhibition in the case of habituation. Both changes are integrated at the presynaptic level, mediated by changes in $\mathrm{Ca}^{2+}$ influx in response to the action potential. In habituation, $\mathrm{Ca}^{2+}$ influx is decreased into the sensory neuron terminal, resulting in depression of glutamate release. In sensitization, on the other hand, the activity of a facilitating serotonergic interneuron induces phosphorylation of a $\mathrm{K}^{+}$channel and lengthening of the depolarization, thus increasing the influx of $\mathrm{Ca}^{2+}$ and the release of glutamate (facilitation; 1,7 ).

Aplysia also exhibits a more complex form of associative learning, typical of higher animals, known as classical conditioning. In this learning paradigm, the animal is given a strong and painful unconditioned stimulus (that if administered alone would produce sensitization) in association with a weak, innocuous, conditioned stimulus (that if administered alone would produce habituation). Following the repeated pairing of these two stimuli over the trials, the animal learns to associate them and to react to the isolated conditioned stimulus with an enhanced response (greater than sensitization to the noxious stimulus). Classical conditioning is reflected in the neural circuitry as a greatly enhanced synaptic strength of the input connec- tions between the sensory neuron and the motor neuron. In contrast to non-conditioned learning, this potentiation involves both presynaptic and postsynaptic mechanisms. The coincidence of the two stimuli is revealed by specific coincidence detectors located on both sides of the synapse [1,7]. This simple model, highly conserved phylogenetically, tells us that association of events, sensed by synaptic coincidence detectors, generates long-term synaptic changes (such as LTP) that maintain the associative memory over time.

The studies on the mechanisms involved in explicit memory are more complex, as explicit memory involves conscious recall and the integration of multiple sensory inputs. Thus, these studies are not feasible in invertebrates and lower vertebrates, but instead require the complexity of the mammalian nervous system. Experimental work in rodents and man has provided strong evidence for the involvement of the hippocampus in many kinds of explicit memory, and particularly in spatial memory. The famous case of Henry Gustav Molaison (the patient H.M.; 1926-2008), who underwent a bilateral hippocampectomy in 1953 to cure an intractable form of epilepsy, first revealed that in the absence of the hippocampus we loose the ability to consolidate new explicit memories (Figure 2). Interestingly, in H.M., short-term memory, old explicit memories and implicit memory processes were completely normal. The intuition by Brenda Milner on the role of hippocampus in the consolidation of new explicit memories was later confirmed by functional MRI studies, which demonstrated an activation of the medial temporal lobe in all tasks in which the subject memorizes a map or mentally rehearses an itinerary. Moreover, studies on the rodent hippocampus have revealed the existence of "place cells", whose firing is primarily controlled by the position of the animal and by distant visual cues that create an internal representation of the animal's location with respect to the surrounding environment [17].

The key role of the hippocampus in the formation of new explicit memories and the fact that this brain area exhibits the most known and extensively studied form of synaptic plasticity, namely long-term potentiation (LTP), has directed research towards the demonstration of a direct link between LTP and memory $[1,7,17,18]$. A large number of studies have demonstrated that LTP is indeed a valid model of "memory storage": hippocampal LTP can be induced by animal experience and, conversely, conscious learning is impaired under conditions in which LTP is impaired or abolished. LTP has all the features required to be the cellular mechanism of explicit memory as it is associative in nature, is triggered by the coincidence of events and can be activated by endogenous patterns of electrical activity (e.g. the $\mathrm{Q}$ rhythm). The molecular mechanisms that mediate the generation of hippocampal LTP are surprisingly conserved across evolution and are closely similar to the mechanisms of associative learning iden- 
tified in invertebrates. Both pre- and post-synaptic mechanisms participate in the early phase of LTP expression, while the late phase of LTP involves activation of transcription factors and regulation of transcription of the target genes [14,19,20-24].

Memory needs time to be stabilized in the hippocampus before the final storage. In fact, LTP induced by an experience is inhibited by a novel experience administered soon after the first one (interference), whereas an LTP established for more than one hour is immune to this reversal mechanism. These observations suggest that the critical event in determining the retention of information may consist in the stabilization of the potentiated hippocampal synapses in order to resist to LTP reversal upon new information [19]. Although the hippocampus is fundamental to the acquisition of new memories, it appears to be dispensable after the memory has been fully consolidated. Although patient H.M.
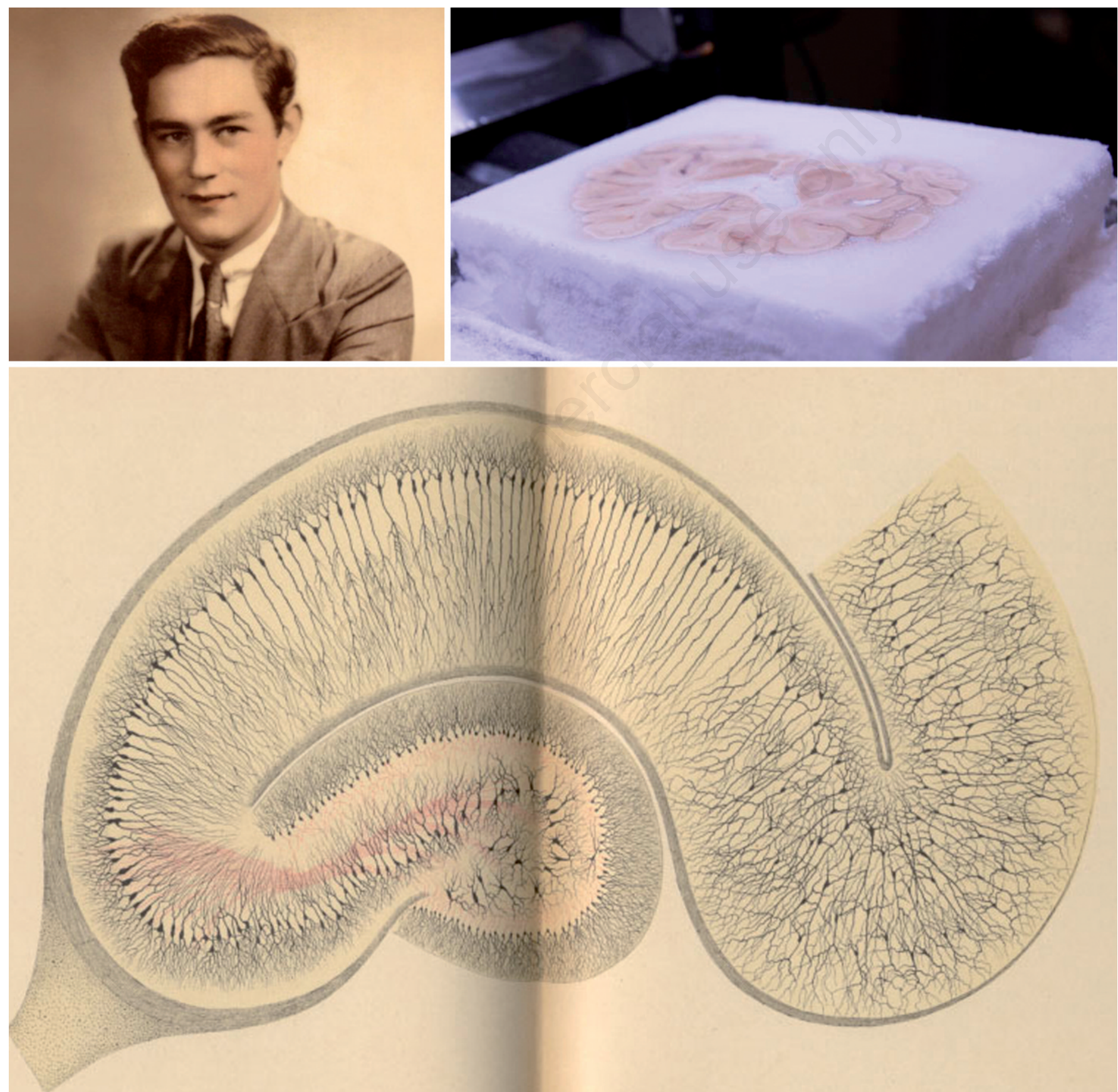

Figure 2. Upper panels: Henry Gustav Molaison (the patient H.M.; 1926-2008) and preparation of sections of his brain embedded in paraffin by the Brain Observatory at the University of California San Diego. Lower panel: The hippocampal formation stained by the "reazione nera" as it appears in the original drawings by Camillo Golgi (1843-1926). 
was totally unable to lay down new memories, he was still able to remember his past life preceding the bilateral ablation of the hippocampi. This indicates that permanent memories are distributed among different cortical regions according to the various perceptual features ("feature map") and that these various aspects are linked in a "master map" so that, upon recall, the different components of a memory are bound together to reproduce the memory in its integrity [25]. It is currently believed that this memory transfer process occurs largely during sleep, particularly REM sleep $[7,14]$. Thus, the hippocampus may represent both the site of the imprinting of the memory and the temporary store for this trace during the progressive formation of neocortical memory representations [6].

\section{RECALL AND RECONSOLIDATION}

"Life is not what one lived, but what one remembers and how one remembers it in order to recount it. The heart's memory eliminates the bad and magnifies the good; and thanks to this artifice we manage to endure the burdens of the past."

Gabriel Garcia Marquez (1927)

Upon new learning, a short-lived memory (short-term memory, STM) is formed that can be either stabilized over the following several hours or pruned out. If this consolidation process takes place, a long-term memory (LTM) is formed that is thought to be rather stable over time and stored as permanent modifications in the wiring of the brain in modality-specific areas. Traditionally, consolidation has been considered as an event occurring only once in the biological history of a memory, and recall of a given memory has been often considered a good exercise against forgetting. However, it has only recently become clear that retrieval does not directly reinvigorate memories, rather it makes them return to a labile state susceptible to disruption and interference which needs further consolidation (the so-called reconsolidation process). In the "Invisible Cities" (1993), Italo Calvino wrote: "Memory's images, once they are fixed in words, are erased," Polo said. "Perhaps I am afraid of losing Venice all at once, if I speak of it, or perhaps, speaking of other cities, I have already lost it, little by little."

This process, originally proposed in the 1960s [26], has been recently elucidated. Reconsolidation appears to be a highly dynamic process that occurs every time memories are reactivated. From a general point of view, consolidation and reconsolidation should be considered as part of the fundamental process of memory stabilization that allows a memory to be preserved, recalled and refreshed over the years. It is commonly found that memories do not remain unchanged over time, but that they undergo transformations in their basic elements and emotional content that have nothing to do with fading. This phenome- non implies that consolidation that is carried out soon after the salient experience cannot be the unique mechanism involved. Rather, reconsolidation provides a dynamic mechanism for updating and modifying memories while they are recalled. What is counterintuitive in this general scheme of memory processes is that a stable memory goes back to a labile state when it is recalled, that is to say that memory recalling is per se an amnesic challenge. However, as memories are not printed as tracks in a compact disk, but are dynamically stored as changes in activity patterns of neuronal networks (which in turn depend on modulation of synaptic strength), it is understandable that reactivation of these activity patterns during recall may change the plastic substrate of the memory, so that additional plasticity changes are needed to preserve it (Figure 3). Moreover, the temporal dynam-
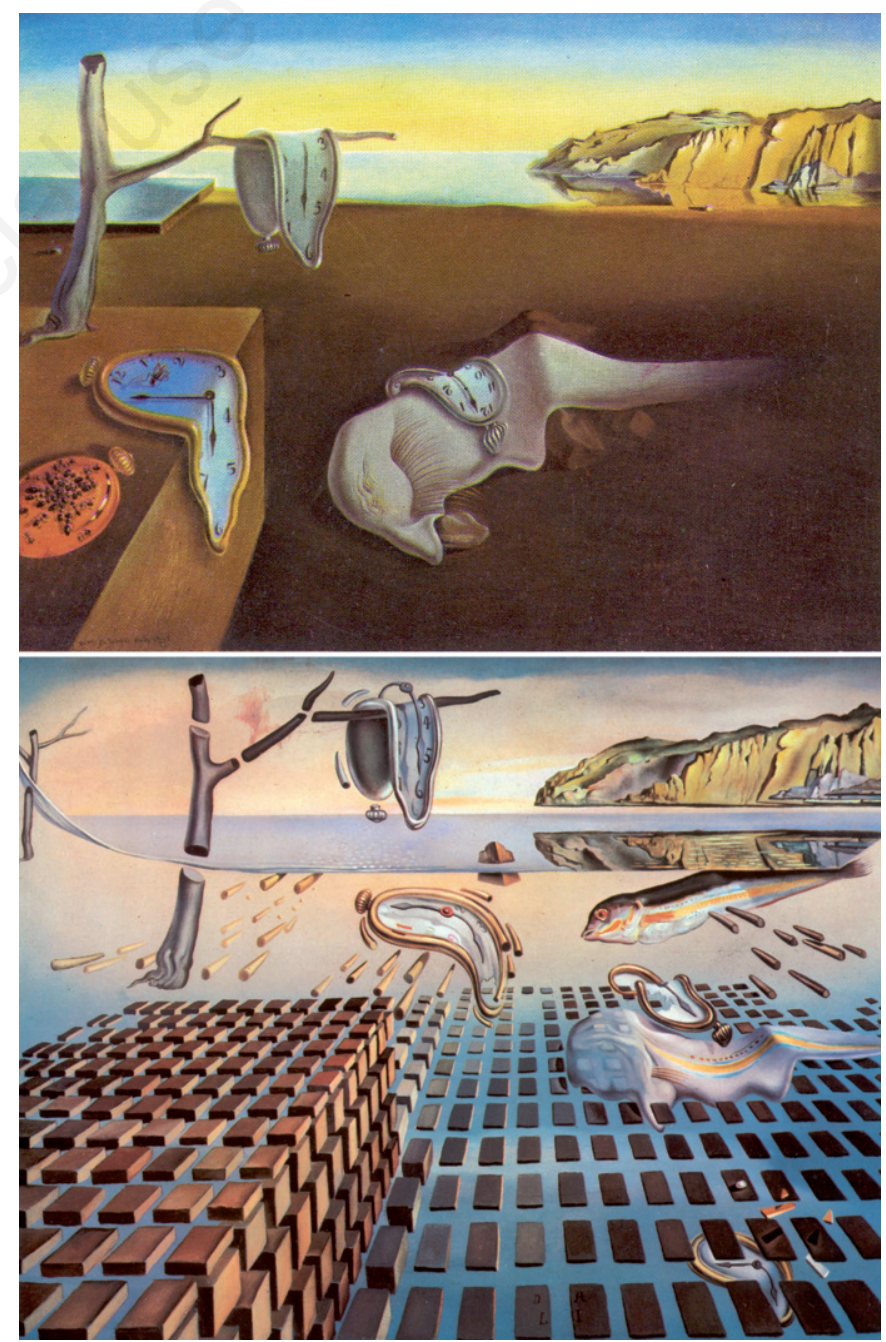

Figure 3. Art representation of memory consolidation and forgetting. The Salvador Dalì's masterpieces "The persistence of memory" (1931) and "Disintegration of the persistence of memory" (1952) suggest that consolidated and reconsolidated memories change and tend to fade with time. 
ics and the extent by which a memory is deconsolidated upon retrieval strongly depend on the strength of the initially consolidated trace, on the intensity of reactivation and on the number of reactivation episodes over time. As a general rule, stronger memories are less susceptible to forgetfulness, and the stronger the reactivation the more labile the memory becomes. It is as if reactivation subtracts part of the memory body and the subtracted part has to be "rebuilt" by reconsolidation. Interestingly, although every time a memory is reactivated it regresses to a labile state and needs reconsolidation, each successive reactivation task requires a progressively smaller reconsolidation. The memory therefore becomes rather stable after several cycles, and successive retrieval episodes will not disrupt the trace; the respective reconsolidation episodes will only modify it [27-29].

As mentioned above, Consolidation (i.e. the transformation of STM into LTM) requires transcription of specific genes and protein synthesis and involves an array of highly conserved signalling pathways that collectively render the memory resistant to cell turnover. Distinct areas are engaged over time in a precise temporal and spatial sequence at both cellular and systems levels. It is well-known that hippocampus-related memories are hippocampus-dependent only over a limited period and that at later times they become hippocampus-independent (remote memories), indicating that other brain regions connected to the hippocampus have undergone a sequential memory imprinting [28,30].

As mentioned above, when the memory is reacti- vated, it regresses to an STM labile state (post-reactivation STM or PR-STM) that is again hippocampus dependent and sensitive to interference (Figure 4). Thus, the memory needs to be reconsolidated in order to become "post-reactivaton" LTM (PR-LTM). However, if the memory is not reactivated, it will remain in a stable state that will slowly fade away over time. Thus, recall appears to disrupt this process of slow decay of LTM. While the exact mechanisms of recall are not fully understood, reconsolidation has been thoroughly studied by the use of transcription or translation inhibitors or of genetically altered mice lacking specific proteins involved in synaptic plasticity. The most conservative mechanism that can be envisaged for memory reconsolidation is that this process employs the very same molecular mechanisms used for consolidation of STM and that both cellular and systems reconsolidation processes occur. Under conditions of inhibition of protein synthesis, the functional and structural changes that mediate LTM become either dysfunctional or actively removed in 4-24 hours after reactivation $[14,30]$.

Reconsolidation has been found to occur in many species from invertebrates to vertebrates (including mammals) and therefore represents a highly conserved fundamental process in memory storage. To give an example, let us have an animal subjected to a classical conditioning trial that, after the memory is fully stabilized, is exposed to the conditioning stimulus to reactivate the memory. If the animal is treated with anisomycin in the reactivation session, it exhibits an intact PR-STM, but the PR-LTM is markedly impaired. Interestingly, if the

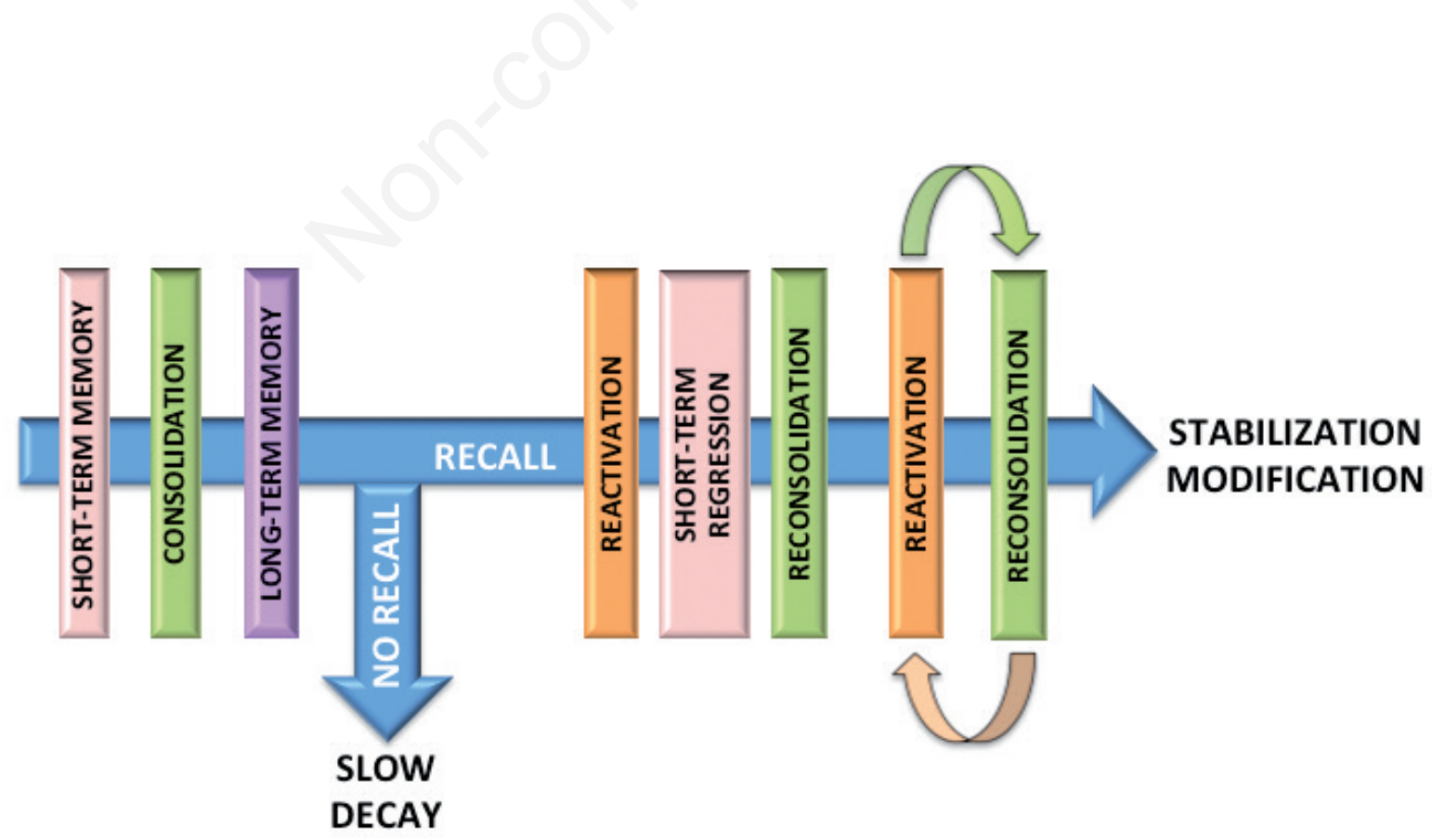

Figure 4. Lifetime and progressive transformations of memory traces. Once short-term memories have been consolidated into long-term memories, they will slowly fade away, if not recalled. On the contrary, recalled long-term memories destabilize and need a process of reconsolidation to be preserved. Successive cycles of recall and reconsolidation make the memory more stable but, at the same time, rearrange and progressively modify the memory trace. 
animal is not challenged for memory reactivation, protein inhibition is ineffective on the LTM acquired in the conditioning session, demonstrating that only the reactivated memory becomes sensitive to disruption, unless synthesis of new proteins is allowed.

The sensitivity to protein inhibition applies only to a narrow time window: if the anisomycin "amnesic" treatment is administered several hours after reactivation it is ineffective, indicating that reconsolidation, like consolidation, is a time-dependent mechanism and that the time needed for reconsolidation is generally shorter that that needed for consolidation. This picture was observed in the case of diverse memory paradigms, including contextual or fear conditioning, passive avoidance, object recognition, taste aversion, motor sequence learning, etc. As for consolidation, reconsolidation is not demonstrated only in behavioural tasks, but it also has neurophysiological correlates. It has been demonstrated that if anisomycin is given 2 hours after LTP induction, it does not affect LTP maintenance. The most conservative explanation is that both consolidation and reconsolidation share the same mechanisms of synaptic rearrangement and permanent tuning of the strength of synaptic connections. Indeed, this seems to be often the case, although similarities between the two processes are not complete and important differences exist in either the molecular actors involved or in the target brain regions or both $[14,28,30]$.
Recent data have shown that in humans existing declarative memories can be selectively rewritten by disrupting reconsolidation using a noninvasive retrieval-relearning technique. This reconsolidation-associated amnesia can be achieved 48 hours after formation of the original memory, but only if relearning occurs soon after retrieval [31]. These results demonstrate that also declarative memories, whose encoding and retrieval rely on a distributed network, are susceptible to reactivation-induced lability, and that memory impairment can be obtained in a behavioral paradigm, without the use of pharmacological agents.

In conclusion, memory recall destabilizes LTM and poses the need for memory reconsolidation. Successive cycles of reconsolidation make the memory more stable, even in the presence of successive retrievals and, at the same time, rearrange and slowly modify the trace of the memory so that the most salient and emotionally significant features are preserved or even enhanced (Figure 4). Consolidation and reconsolidation often occur in distinct brain regions or subregions, consistent with the idea that consolidated memories are sorted to diverse brain areas, but the molecular mechanisms involved largely overlap.

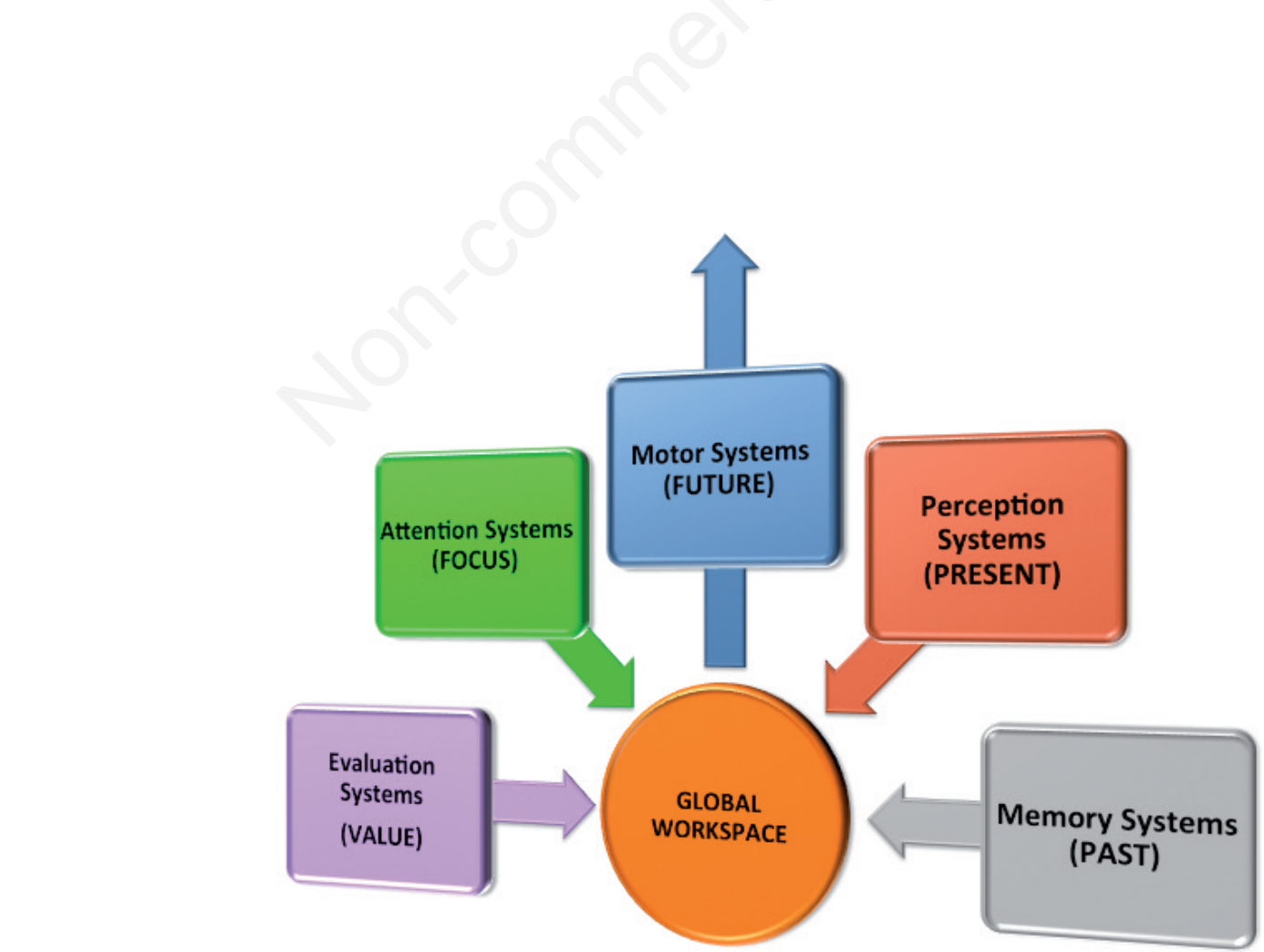

Figure 5. Integration of past, present and future in the global workspace. Five main specialized elaborators (perception, attention, evaluation, memory, motor output), while working autonomously, are strictly interconnected by long-range intracortical and inter-cortical neurons and form a global workspace that is thought to be the neural correlate of consciousness. In this workspace, the scanning of time is represented by memory systems (the past), perceptive systems (the present) and motor systems (the future) [32,33]. 


\section{MEMORY AND THE EXPERIENCE OF TIME}

Time and space are central features of our existence and our brain needs to build a reference system to orient the individual in the four dimensions. Remembering the past or imagining our future are both "time travels" and "time experiences". In addition, over our life, the present time window that separates past and future continuously moves ahead, so that the ratio between future and past progressively decreases as we get old.

Time, for our brain, is a generalization of successions of events that we recognize by applying spatial criteria to temporal sequences. Each event in our life is positioned along the time axis in association with other events of our emotional, working and social life, as if it were a film editing putting together short clips of our life (autobiographical or episodic memory). We often switch between space and time and time is often used to quantify a spatial distance (e.g. the light year, the time to cover a mountain trail, etc.). We can say that memory is our past; memory assists our present, by giving us safety and sense of individuality; and finally that memory is our future, by allowing our mind to trace a thin line between past and future and measure the relentless flowing of time of our life. Memory is one of the high order computational systems that compose the cortical global workspace from which our consciousness is thought to emerge $[32,33]$. The five main elaborators, working autonomously in the field of perception, attention, evaluation, memory, motor output are strictly interconnected by long-range intra-cortical and inter-cortical neurons. In this workspace, the sense of time relies on the memory systems, representing the past, the perceptive systems, representing the present, and the motor systems, representing the future (Figure 5).

How fundamental is memory to build up our ability to imagine and navigate the future? The ability to imagine future scenarios (the episodic thought of the future) seems to be directly related to our ability to recall past experiences (our episodic memory), and involves sampling of our memories [34]. Episodic memory emerges in children at the age of 4-5 years; in parallel, also the ability of projecting oneself into the future emerges approximately at the same age, in concert with the ability to vividly recollect past events [35]. Amnesic patients, who do not remember single episodes from the past, cannot project themselves into the future. Although they understand the concept of time, in the absence of memories of the past, they cannot undergo a mental time travel in the future [36]. Patients affected by severe depression experience difficulties in recalling past episodes of their life; by the same token, they are also unable to engage episodic future thoughts. Such inability to envision a possibly brighter "future" could play a role in maintaining the depressive state [34].

In fMRI studies, brain regions essential for reinstating past experiences also play a fundamental role in constructing future autobiographical episodes. These brain regions include the occipital cortex, the posterior cingulate cortex and the medial temporal cortex. These findings indicate that individuals, in order to generate and effective and plausible image of the future, have to
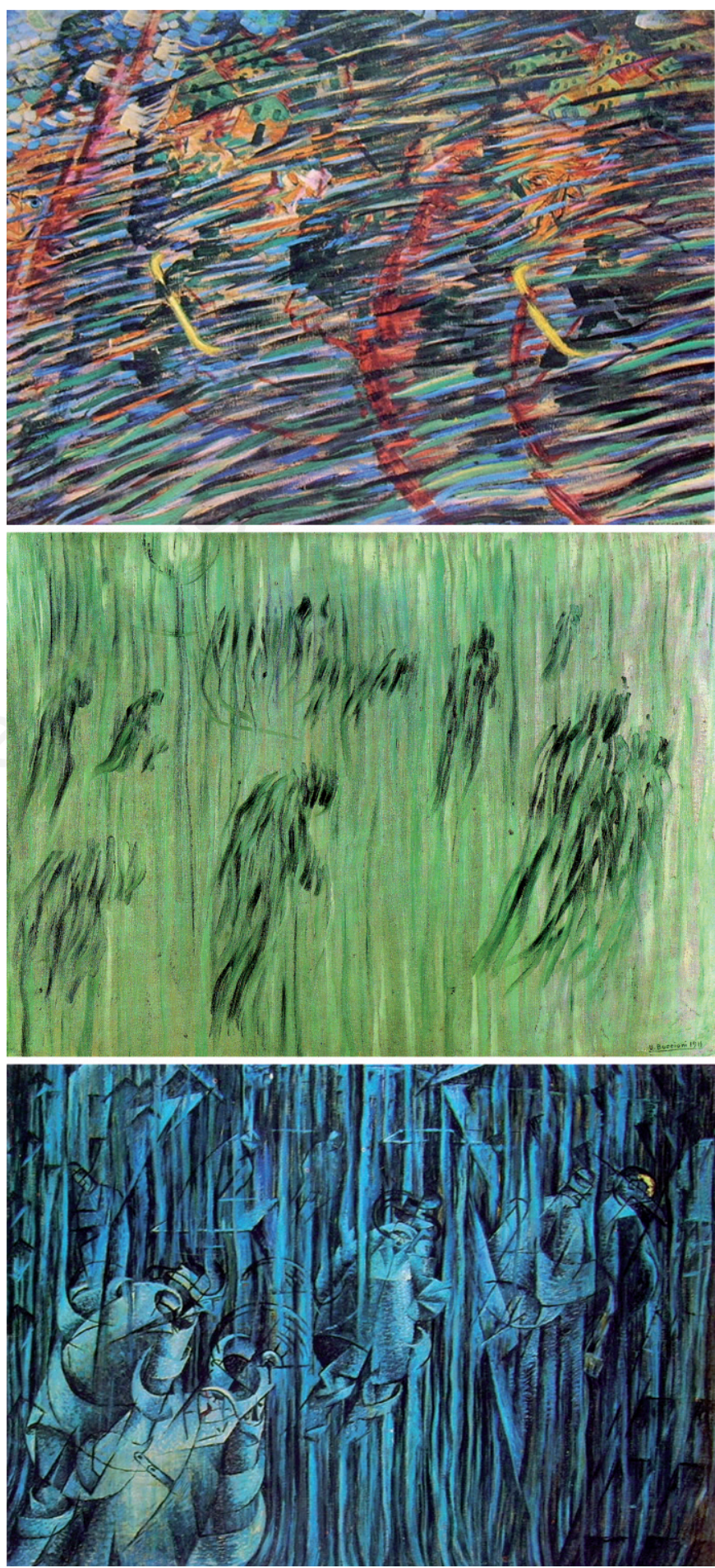

Figure 6. The perception of space and time in our mind is beautifully given in the Umberto Boccioni's painting series "States of Mind": "Those who go" (States of Mind I, 1911; top), " Those who stay " (Study, 1911; middle), "Those who stay" (States of Mind II, 1911; bottom). 
reactivate images and personal events belonging to their past. In addition, further brain areas are recruited when mentally rehearsing a future behavioral sequence such as the lateral premotor cortex, the posterior cerebellum and the hippocampus $[37,38]$.

The intricate overlap between memory, past and future is poetically described in this inspired piece by the Italian writer Italo Calvino (Invisible Cities, 1993) about the city of Isidora: "When a man rides a long time through wild regions, he feels the desire for a city. Finally he comes to Isidora, a city where the buildings have spiral staircases encrusted with spiral seashells, where perfect telescopes and violins are made, where the foreigner hesitating between two women always encounters a third, where cockfights degenerate into bloody brawls among the bettors. He was thinking of all these things when he desired a city. Isidora, therefore, is the city of his dreams: with one difference. The dreamed-of city contained him as a young man; he arrives at Isidora in his old age. In the square there is the wall where the old men sit and watch the young go by; he is seated in a row with them. Desires are already memories." In Isidora, the visitor's expectations for the future appear to him as memories (Figure 6).

\section{CONCLUSIONS}

"You have to begin to lose your memory, if only in bits and pieces, to realize that memory is what makes our lives. Life without memory is no life at all, just as an intelligence without the possibility of expression is not really an intelligence. Our memory is our coherence, our reason, our feeling, even our action. Without it, we are nothing."

Luis Buñuel (1900-1983)

Memory is not just an ability to store information, but the essence of our beings, the basis of our individuality and our consciousness. Each individual knows that he/she is unique, not merely because of his/her external appearance, but because of his/her personal history, behavior and ability to face daily life. Learning and memory are achieved by permanently shaping neuronal circuits and inter-neuronal connections. These modifications are initially labile, but if they are perceived as useful and salient, then they are consolidated and eventually reconsolidated to become a stable memory. However, most of the percept is quickly discarded, in a process of selection: what we think is relevant is remembered, although it tends to fade and change with time. Umberto Eco, in The Mysterious Flame of Queen Loana, wrote: “...memory acts like a convergent lens in a camera obscura: it focuses everything, and the image that results from it is much more beautiful than the original". Although many questions remain open, the astonishing progress in the field of molecular and cellular neuroscience is greatly contributing to the understanding of the exact role of gene products and signaling pathways in distinct processes of memory and forgetting, and it is likely that within a few years this knowledge will be translated into the development of novel therapeutic approaches to memory disorders.

\section{REFERENCES}

1. Kandel ER, Schwartz JH, Jessell TM, Siegelbaum SA, Hudspeth AJ. Principles of neural sciences. New York: McGraw Hill Professional; 2012.

2. Hebb DO. Organization of behavior: a neuropsychological theory. New York: John Wiley and Sons; 1949.

3. Freud S. The Standard Edition of the Complete Psychological Works of Sigmund Freud. London: Hogarth Press; 1953.

4. Sejnowski TJ. The book of Hebb. Neuron 1999;24:773-6.

5. Centonze D, Siracusano A, Calabresi P, Bernardi G. The Project for a Scientific Psychology (1895): a Freudian anticipation of LTPmemory connection theory. Brain Res Rev 2004;46:310-4.

6. Hasselmo ME, McClelland JL. Neural models of memory. Curr Opin Neurobiol 1999;9:184-8.

7. Kandel ER, Pittenger C. The past, the future and the biology of memory storage. Philos Trans R Soc Lond B Biol Sci 1999;354: 2027-52.

8. Silva AJ, Josselyn SA. The molecules of forgetfulness. Nature 2002;418:929-30.

9. Greengard P, Valtorta F, Czernik AJ, Benfenati F. Synaptic vesicle phosphoproteins and regulation of synaptic function. Science 1993;259:780-5.

10. Greengard P. The neurobiology of slow synaptic transmission. Science 2001;294:1024-30.

11. Elgersma $Y$, Silva AJ. Molecular mechanisms of synaptic plasticity and memory. Curr Opin Neurobiol 1999;9:209-13.

12. Abel T, Lattal KM. Molecular mechanisms of memory acquisition, consolidation and retrieval. Curr Opin Neurobiol 2001;11:180-7.

13. Genoux D, Haditsch $U$, Knobloch $M$, et al. Protein phosphatase 1 is a molecular constraint on learning and memory. Nature 2002;418:970-5.

14. Alberini CM. Mechanisms of memory stabilization: are consolidation and reconsolidation similar or distinct processes? Trends Neurosci 2005;28:51-6.

15. Bozon B, Kelly A, Josselyn SA, et al. MAPK, CREB and zif268 are all required for the consolidation of recognition memory. Philos Trans R Soc Lond B Biol Sci 2003;358:805-14.

16. Blackemore C. Mechanics of the mind. Cambridge: Cambridge University Press; 1977.

17. Colgin LL, Moser EI, Moser MB. Understanding memory through hippocampal remapping. Trends Neurosci 2008;31:469-77.

18. Moser EI, Paulsen O. New excitement in cognitive space: between place cells and spatial memory. Curr Opin Neurobiol 2001;11:745-51.

19. Miller S, Mayford M. Cellular and molecular mechanisms of memory: the LTP connection. Curr Opin Genet Dev 1999;9:333-7.

20. Matynia A, Kushner SA, Silva A. Genetic approaches to molecular and cellular cognition: a focus on LTP and learning and memory. Annu Rev Genet 2002;36:687-720.

21. Alberini CM. The role of protein synthesis during the labile phases of memory: revisiting the skepticism. Neurobiol Learn Mem 2008;89:234-46.

22. Alberini CM. Transcription factors in long-term memory and synaptic plasticity. Physiol Rev 2009;89:121-45.

23. Won J, Silva AJ. Molecular and cellular mechanisms of memory allocation in neural networks. Neurobiol Learn Mem 2008;89:285-92.

24. Miyashita T, Kubik S, Lewandowski G, Guzowski JF. Networks of 
neurons, networks of genes: an integrated view of memory consolidation. Neurobiol Learn Mem 2008;89:269-84.

25. Seung HS. Reading the book of memory: sparse sampling versus dense mapping of connectomes. Neuron 2009;62:17-29.

26. Lewis DJ. Psychobiology of active and inactive memory. Psychol Bull 1979;86:1054-83.

27. Dudai $Y$, Eisenberg M. Rites of passage of the engram: reconsolidation and the lingering consolidation hypothesis. Neuron 2004;44:93-100.

28. Nader K. Memory traces unbound. Trends Neurosci 2003;6:65-72.

29. Nader K. Re-recording human memories. Nature 2003;425:571-2.

30. Nader K, Hardt $O$. A single standard for memory: the case for reconsolidation. Nat Rev Neurosci 2009;10:224-34.

31. Chan JCK, LaPaglia JA. Impairing existing declarative memory in humans by disrupting reconsolidation Proc Natl Acad Sci USA 2013;110:9309-13.

32. Dehaene S Kerszberg M Changeux JP. A neuronal model of a global workspace in effortful cognitive tasks. Proc Natl Acad Sci USA 1998;95:14529-34.

33. Baars BJ. A cognitive theory of consciousness. Cambridge: Cambridge University Press; 1998.

34. Szpunar KK, McDermott KB, eds. Remembering the past to imagine the future. In: Cerebrum: emerging ideas in brain science. New York: Dana Press; 2008. pp 17-27.

35. Busby J, Suddendorf T. Recalling yesterday and predicting tomorrow. Cogn Dev 2005;20:363-72.

36. Klein SB, Loftus J, Kihlstrom JF. Memory and temporal experience: the effects of episodic memory loss on an amnesic patient's ability to remember the past and imagine the future. Soc Cogn 2002;20:353-79.

37. Szpunar KK, Watson JM, McDermott KB. Neural substrates of envisioning the future. Proc Natl Acad Sci USA 2007;104:642-7.

38. Addis DR, Wong AT, Schacter DL. Remembering the past and imagining the future: common and distinct neural substrates during event construction and elaboration. Neuropsychologia 2007;45:1363-77. 